zwischen Religion und Wirtschaft im Gefolge der Luhmannschen Systemtheorie als Gedanken erwähnt, der sich in dieser Hinsicht weiter ausführen ließe (vgl. S. 380 ff.). Die monierte Trennschärfe zur abgelehnten These „Geld als Gott“ müsste hierfür allerdings entweder spezifiziert oder aufgegeben werden.

Funding Open Access funding enabled and organized by Projekt DEAL.

Open Access Dieser Artikel wird unter der Creative Commons Namensnennung 4.0 International Lizenz veröffentlicht, welche die Nutzung, Vervielfältigung, Bearbeitung, Verbreitung und Wiedergabe in jeglichem Medium und Format erlaubt, sofern Sie den/die ursprünglichen Autor(en) und die Quelle ordnungsgemäß nennen, einen Link zur Creative Commons Lizenz beifügen und angeben, ob Änderungen vorgenommen wurden.

Die in diesem Artikel enthaltenen Bilder und sonstiges Drittmaterial unterliegen ebenfalls der genannten Creative Commons Lizenz, sofern sich aus der Abbildungslegende nichts anderes ergibt. Sofern das betreffende Material nicht unter der genannten Creative Commons Lizenz steht und die betreffende Handlung nicht nach gesetzlichen Vorschriften erlaubt ist, ist für die oben aufgeführten Weiterverwendungen des Materials die Einwilligung des jeweiligen Rechteinhabers einzuholen.

Weitere Details zur Lizenz entnehmen Sie bitte der Lizenzinformation auf http://creativecommons.org/ licenses/by/4.0/deed.de.

\title{
Willke, Helmut: Komplexe Freiheit. Konfigurationsprobleme eines Menschenrechts in der globalisierten Moderne, 308 S., transcript, Bielefeld 2019.
}

\section{Florian Eyert · Florian Irgmaier}

Online publiziert: 28. Juli 2020

(C) Der/die Autor(en) 2020

Freiheit und ihre politischen Bedingungen sind in den letzten Jahren erneut zu einem zentralen Gegenstand sozialwissenschaftlicher Auseinandersetzung geworden. Allerorts lassen sich Gefährdungen des Liberalismus ausmachen, vom Populismus über

F. Eyert $(\bowtie) \cdot$ F. Irgmaier

Weizenbaum-Institut für die vernetzte Gesellschaft/Wissenschaftszentrum Berlin für Sozialforschung, Berlin, Deutschland

E-Mail: florian.eyert@wzb.eu

F. Irgmaier

E-Mail: florian.irgmaier@wzb.eu 
die illiberale Demokratie bis hin zum Autoritarismus. Unter der Fülle vorliegender analytischer Zugänge zur gegenwärtigen Lage der Freiheit nimmt Helmut Willkes ambitionierte Studie „Komplexe Freiheit“ eine Sonderposition ein, denn sie geht gesellschaftsanalytisch und modernitätstheoretisch und damit genuin soziologisch vor. Willke zielt mit ihr auf nicht weniger als den Entwurf eines neuen Verständnisses von Freiheit, das den Herausforderungen der komplexen Gegenwartsgesellschaft durch eine Rekonfiguration des Freiheitsbegriffs sowie eine Rekonstruktion seiner empirischen Verwirklichungsbedingungen begegnet.

Der Autor identifiziert zunächst zwei übergreifende Entwicklungen, die einen neuen Freiheitsbegriff notwendig erscheinen lassen: Erstens verlören im Zuge der Globalisierung Nationalstaaten - für Willke die Garanten von Freiheit, sofern sie demokratisch verfasst sind - an Souveränität, zum einen durch supranationale Organe, zum anderen durch globale systemische Interdependenzen. Zweitens beeinträchtige die Digitalisierung, die in erster Linie als „Wirbelsturm digitaler Datenströme“ (S. 88) erscheint, Freiheitspotenziale sowohl durch die Zunahme digitaler Spuren und damit verbundener Möglichkeiten der Verhaltenssteuerung als auch durch die subjektiv als Datenflut erlebte Ausweitung von Handlungsoptionen. Auf den Begriff gebracht werden beide Entwicklungen durch ihre radikale Steigerung von Komplexität und Kontingenz. Diese produzierten neuartige Bedrohungen für die Freiheit, die Willke im Kern in einem Wandel von Zwang zu Manipulation identifiziert, zugleich aber weiter engführt: „Die eigentliche Begrenzung von Freiheit“, so heißt es gleich zu Beginn, „folgt aus Nichtwissen“ (S. 12). Vor diesem Hintergrund macht sich der Autor daran, die Bedingungen eines den beobachtbaren Umständen gerecht werdenden Verständnisses von Freiheit zu identifizieren. Dieses ist nicht mehr monolithisch angelegt - „Freiheit writ large“ (S. 279) -, sondern vielmehr partiell, dezentriert und in einem verteilten Zusammenspiel gedacht. Konstruiert ist es entlang zweier Dimensionen. In der Dimension der gesellschaftlich-demokratischen Suprastruktur setzt Willke auf neue Mechanismen der horizontalen Subsidiarität und Föderalität. Die Steuerung komplexer Systeme sei auf Ansätze verteilter Expertise angewiesen und Kontingenzkontrolle durch Kontextsteuerung zu bewerkstelligen. In der Dimension der individuell-informationellen Tiefenstruktur schlägt der Autor eine Aktualisierung der klassischen Mill'schen Trias von Willens-, Entscheidungsund Handlungsfreiheit vor, die auf eine Gewährleistung von Meinungs-, Wahl- und Kommunikationsfreiheit abstellt. In der Konsequenz empfiehlt Willke dann vor allem die Stärkung von institutionellen Lernprozessen, die, wenn antizipativ und proaktiv gestaltet, eine Entgegnung auf das Problem der Komplexität darstellen könnten.

Zwei grundlegende Mängel der Argumentation möchten wir an dieser Stelle hervorheben. Der erste betrifft das Design der Begriffsarbeit. Diesbezüglich fällt zunächst das Fehlen systematischer methodologischer und methodischer Vorüberlegungen auf. Eine Arbeit, die einen ,der gesellschaftlichen Komplexität angepasste[n] und insofern adäquat komplexe[n] Begriff von Freiheit" (S. 8) erarbeiten soll, müsste bereits vor der konkreten Auseinandersetzung mit Freiheitsbegriffen wichtige Fragen klären, etwa, was unter einem Begriff verstanden werden und was der zu entwickelnde Freiheitsbegriff leisten soll. Zwar lässt sich leicht ersehen, dass Willke eine Begriffsarbeit in kritischer Absicht anstrebt, also einen überzeugenden Maßstab zur Beurteilung gesellschaftlicher Verhältnisse und Handlungsoptionen entwickeln will; 
doch wären die Konturen des gesuchten Freiheitsbegriffs klarer, wenn vor Beginn der Untersuchung die abstrakten Merkmale eines brauchbaren kritischen Freiheitsbegriffs geklärt wären. Unbeantwortet bleibt auch die Frage nach dem Verhältnis von Begriff und Praxis: Welche sind die allgemeinen Bedingungen, nach denen entschieden werden kann, ob ein konkreter normativer Begriff einer gesellschaftlichen Situation angemessen ist? Wie Theoriefiguren der Kontrafaktizität, etwa der regulativen Idee bei Immanuel Kant oder der normativen Erwartungen in der Systemtheorie verdeutlichen, ist die empirische Nichtrealisierung eines Begriffsinhalts noch kein prinzipielles Argument gegen den Begriff selbst - die empirischen Unfreiheiten des 20. Jahrhunderts haben den liberalen Freiheitsbegriff keineswegs desavouiert. $\mathrm{Zu}$ Recht zielt Willke deshalb, wie sein Vorgehen zeigt, auf die Entwicklung eines Freiheitsbegriffs, der unter den Prämissen seiner soziologischen Theoriearbeit als prinzipiell realisierbar erscheint. Dennoch hätte ein reflexiverer Umgang mit diesem Aspekt von Begriffsarbeit der Untersuchung eine tragfähigere Struktur verleihen und die Überzeugungskraft von Willkes Freiheitskonzeption stärken können. Dies gilt umso mehr, als es sich bei dem in der Systemtheorie sonst unüblichen Begriff der Freiheit um ein essentially contested concept handelt, dessen Bestimmung einen inhärent politischen Charakter hat und somit nur in begrenztem Maße durch soziologische Theoriearbeit zu leisten ist.

Auffallend sind zweitens die zeitdiagnostischen Aspekte der Analyse, die vor allem bezüglich der Digitalisierung mitunter deutlich hinter dem Stand der Forschung zurückbleiben. So tritt die umfangreiche wissenschaftliche und insbesondere soziologische Literatur zur Digitalisierung in weiten Teilen zugunsten eher essayistischer und feuilletonistischer Quellen in den Hintergrund. Fragen des immerhin mit dem Ziel der Bewahrung von Selbstbestimmungs- und Freiheitspotenzialen antretenden Datenschutzes spielen dabei eine ebenso geringe Rolle wie die wachsende Bedeutung KI-gestützter Überwachungsmethoden oder die Entwicklung neuartiger, digitaler Proteststrategien. So erscheint dann die tatsächliche empirische Zeitdiagnose des Buches bisweilen eindimensional - etwa wenn die Digitalisierung als eine Technologie bezeichnet (S. 74) wird, die sich ,flächendeckend“ umsetzen ließe (S. 77) und wird der Komplexität des höchst heterogenen Prozesses der digitalen Transformation mit seinen zahlreichen Schauplätzen und Konfliktachsen mit Blick auf seine Freiheitsimplikationen nicht gerecht.

Trotz genannter Begrenzungen liefert Willkes Buch wertvolle Anstöße für eine Debatte über die Rolle klassischer politischer Leitbegriffe in einer unübersichtlichen Gegenwart. Die zukünftig zweifellos weiter in die Tiefe gehende soziologische Forschung zu den Spannungsfeldern der Spätmoderne dürfte in ihnen fruchtbare Referenzpunkte für eine kritische Auseinandersetzung finden.

Funding Open Access funding provided by Projekt DEAL.

Open Access Dieser Artikel wird unter der Creative Commons Namensnennung 4.0 International Lizenz veröffentlicht, welche die Nutzung, Vervielfältigung, Bearbeitung, Verbreitung und Wiedergabe in jeglichem Medium und Format erlaubt, sofern Sie den/die ursprünglichen Autor(en) und die Quelle ordnungsgemäß nennen, einen Link zur Creative Commons Lizenz beifügen und angeben, ob Änderungen vorgenommen wurden. 
Die in diesem Artikel enthaltenen Bilder und sonstiges Drittmaterial unterliegen ebenfalls der genannten Creative Commons Lizenz, sofern sich aus der Abbildungslegende nichts anderes ergibt. Sofern das betreffende Material nicht unter der genannten Creative Commons Lizenz steht und die betreffende Handlung nicht nach gesetzlichen Vorschriften erlaubt ist, ist für die oben aufgeführten Weiterverwendungen des Materials die Einwilligung des jeweiligen Rechteinhabers einzuholen.

Weitere Details zur Lizenz entnehmen Sie bitte der Lizenzinformation auf http://creativecommons.org/ licenses/by/4.0/deed.de.

\section{Glatzmeier, Armin: Gerichte als politische Akteure. Zur funktionalen Rolle der Verfassungsgerichtsbarkeit in Demokratien, 555 S., Nomos, Baden-Baden 2019.}

\section{Christoph Gusy}

Online publiziert: 28. Juli 2020

(C) Gesellschaft zur wissenschaftlichen Förderung politischer Literatur e.V. and the Author(s) 2020

Eine wissenschaftlich bislang kaum diskutierte Frage um die Verfassungsgerichtsbarkeit liegt darin: Wie schaffen es die Gerichte, dass die von ihnen kontrollierten Instanzen gefällte Urteile tatsächlich befolgen? Ihr wendet sich das vorliegende Buch im Ansatz zu, dessen Schlusskapitel mit „Stärke und Schwäche im Kontext verfassungsgerichtlicher Kontrollregime" überschrieben ist. Wann ist ein Kontrollregime stark und wann nicht? Und wie lässt sich das messen? Dem soll nicht allein für Deutschland, sondern auch für die anderen EU-Staaten, die Schweiz und die USA nachgegangen werden. Es ist diese Breite des Gegenstandes, welche den besonderen Reiz der Fragestellung ausmacht.

Ausgangspunkt ist, dass Frage und mögliche Antworten nicht allein rechtlich bestimmt sein können. Sie werden zu Recht bei Politik und Politikwissenschaft verortet und abgearbeitet. Am Anfang steht die Explikation der Fragestellung: Allzu unterschiedlich sind die Instanzen, Rechtswege und Verfahren, welche verfassungsgerichtliche Aufgaben erfüllen. Und ebenso heterogen sind kontrollierte Instanzen, Kontrollgegenstände und Kontrollwirkungen. Nur um Verfassungsgerichte geht es hier, die übrigen gerichtlichen Instanzen und Aufgaben werden entgegen dem Titel, aber mit dem Untertitel explizit ausgeklammert. Das bedarf der Abgrenzung

\footnotetext{
C. Gusy $(\bowtie)$

Universität Bielefeld, Bielefeld, Deutschland

E-Mail: christoph.gusy@uni-bielefeld.de
} 